\title{
TEARING INSTABILITY AND THE DEVELOPMENT OF VOIDS IN A LOW-ALLOY STEEL
}

\author{
C. Q. Zheng* and J. C. Radon**
}

North Western Polytechnical University, Xicin. People's Republic of China
Imperial College, London SW7 $2 B X$. England

\section{ABSTRACT}

Ductile fracture tests and static tensile tests were performed on a lowalloy steel BS 4360-50D used extensively in the construction of the large North sea oil rigs. The tearing instability concept was applied to invest igate the fracture process. Ductile fracture occurring by the formation.

Two specific values of plastic strain were noted, namely, $\varepsilon_{p}$ ๓ 0.22

when a large number to the instability At this strain the voids were coalescing and ligaments and partition walls began to fracture.

A linear semi-logarithmic relationship between the relative void volume and the plastic strain was established for smooth cylindrical tensile specimen in the range of necking. Finally, a static toughness value of $J=293 \mathrm{~N} / \mathrm{mm}$ was derived; this compares favourably with experimental results.

\section{KEYWORDS}

Tearing modulus; $J$-integral; ductile fracture; development of voids instability strain.

\section{INTRODUCTION}

Structural safety of very large frameworks such as offshore oil platforms, is of fundamental importance and at the present time a considerable amount of attention is being given to the subject of their construction. The reliability analysis has recently been applied in detail to all designs of oil rigs and in particular to possible fatigue failures. It is well known that these types of large structures are statically and dynamically indeterminate. Consequently, one of the important factors is the redistribution or forces after a partial collapse which can occur only when a 
This force redistribution process is of basic importance for reliable and efficient engineering design. It seems clear that the deformation capacity of any structural member is closely related to the stress-strain properties of the material used. Thus a detailed investigation of these properties and their effect on the mechanical behaviour of the structure is necessary.

A low-alloy steel BS4360-5OD is widely used for the construction of oil rigs and a preliminary investigation of some of its basic properties has been reported (1). This present paper describes three specific aspects of the tensile fracture: namely, the low strain rate tensile test; the vold formation process during the local necking; and the application of the tearing instability concept for tensile cracks under fully plastic conditions.

\section{TEARING MODULUS}

It is a common practice to determine the tensile properties of a material using round bar test-pieces, such as in this investigation, according to the standard BS18 (1971). Static tensile tests were performed at a loading rat of $0.05 \mathrm{~mm} / \mathrm{min}$ on cylindrical specimens, a $=11.28 \mathrm{~mm}$. The specimens were manufactured from a 50 me thick plate with thelr axes parallel to RD. we tesking procedure and the results obtaint are.

After the general yielding and at the beginning of the necking the strain hardening coefficient $n$ reached the value of 0.22 . On completion of the specime was characterised by gross yielding in the gauge length. As discussed later, the voids formed around the inclusions mostly in the necked region. Some voids nucleated before reaching the maximum load, but the majority formed at and after the maximum load. In order to further develop the present understanding of the fracture, the tearing instability criterion $(2,4)$ was applied.

The concept of tearing modulus, $T$, has been developed on the basis of the T-integral resistance curve and the two non-dimensional quantities, $T$ material and $T$ applied. The value of $T$ mat represents all intrinsic properties of the material, while $T_{a p p}$ refers to the geometric configuration of the specimen. Both quantities may be defined as follows:

$$
\begin{gathered}
T_{\text {mat }}=\frac{E}{\sigma_{0}^{2}} \frac{d J m a t}{d a} \\
T_{\text {app }}=\frac{4 L d}{D^{2}}
\end{gathered}
$$

Here the term $T_{\text {app }}$ represents the geometry of a centre-cracked (a penny shape crack) round test-piece or that of a notched round bar. The deformation condition

$$
\begin{aligned}
T_{\text {mat }} & >T_{\text {app }} \ldots \ldots \ldots . . . \text { stable condition } \\
T_{\text {mat }} & <T_{\text {app }} \ldots \ldots \ldots \text { unstable condition }
\end{aligned}
$$

In these expression: $E$ is Young's modulus $\left(2.133 \times 10^{5} \mathrm{MPa}\right.$ for steel), $\sigma_{0}$ is true fracture stress or flow stress (4). a is the relevant crack or flaw size for the stability analysis. Jmat is the value of $J$-integral following the compliance of the machine, grips and the button ends of the specimen are the gauge diameter and the crack length of the specimen respectively.

At the beginning of the necking process no internal crack in the body of the specimen gauge length was assumed,

Therefore:-

$$
\text { i.e. }
$$$$
d=2 a \simeq 0 \text {. }
$$$$
T_{\text {app }} \simeq 0
$$

Using the appropriate experimental data for 50D steel we obtain

$$
T_{\text {mat }}=35.8
$$

The following values $(1,3)$ were applied:

$$
\begin{aligned}
& \frac{d_{J}}{d a}=225 \mathrm{MPa} \\
& \sigma_{0}=1158 \mathrm{MPa}
\end{aligned}
$$

Comparing equations (6) and (7) it is seen that

$$
T_{\text {mat }}>T_{\text {app }}
$$

and consequently, according to equation (3) the condition is stable.

Now considering the point of failure, it will be realised that the situation is different. Four necking tests were performed and the respective values of Tmat. Tapp recorded, resulting in:

$$
T_{\text {mat }}<T_{\text {app }}
$$

this inequality corresponds with the process of unstable deformation, equation (4).

The change in the degree of stability involved in this process may be described as follows. The formation of the neck started at, or close before reaching the maximum load $P_{\max }$, which in the present static monotonic
tests corresponds to the stress $\sigma_{\max }=550 \mathrm{MPa}$. On reaching this load the necking could be easily observed on the surface of the specimen neck. same time a microllaw (a vold) develop latin to grow only after a certain It was assumed that this microflawn. At the same time. Tapp, which until amount of deformation of the specin. now was near zero, started increasing. The formation and be expected that the of the microllaw was a crack $d$ is growing, the value of $T$ app is increasing as well. With the crack d is point where $T$, $=T$, followed by $T$ Tp $>T_{\text {mat }}$ and resulting in the complete separation.

FORMATION OF VOIDS Basically, the micromechanism of ductile fracture takes place in three 
voids; and 3) coalescence of voids leading to final fracture (separation). It has been known for some time that the cohesion of small second phase particles with the matrix is very strong and this applies to nearly al engineering materials. However, the cohesion of larger second phase particles with the matrix is substantially weaker. Consequently, after a certain amount of matrix deformation, some larger particles may crack or Iracture and this process will be followed by the formation of microvoing in low-alloy steels of the type CaS, etc. Due to the rolling processes involved in the manufacture these inclusions are in general ellipsoidal, but as a consequence of the processing they may often be very irregular and also flattened in the transverse directions. This rather complicated geometry of the inclusions strongly affects the deformation behaviour of the material and the prediction of fracture is not an easy task.

In order to investigate the fracture process in more detail the tests were interrupted at convenient intervals. Therefore, in addition to the standar tensile tests described in (1), further tests were performed with suitable interruptions during the yielding process, before necking, during necking, after necking but before instability and finally, at the moment of separat ion. On the completion of these tests all the specimens were split along their longitudinal axes in a liquid nitrogen bath and some of them sectioned by the milling machine and subsequently polished and etched using 28 Nital. The microstructure was studied with the help of the scanning electron microScope JSM-T2OO an scope Jymical formations and growth of voids found in 500 steel:-

Fig. la) A spheroidal inclusion with very small voids.

Fig. 1b) A typical ellipsoidal void with a spheroidal inclusion ( $\varepsilon_{p}$ fo 0.8 ). Fig. 1c) A large number of voids completely enveloping small inclusions.

Some voids nucleated transverse to the loading direction Fig. 2, $\left(\mathrm{CaO} x\left(\mathrm{~A}_{2} \mathrm{O}_{3}\right)_{y}\right.$ in the form of large hexagonal particles.

The void nucleation process was observed typically at the following inclusions. Mns (angular shape, mostly about 10 to 50 m large). Here the void nucleation began at $\varepsilon_{p}<0.15$.

other inclusions, observed in decreasing size, were as follows:

CaS ( $25 \mu \mathrm{m})$.

$\mathrm{CaO} . \mathrm{MgO} . \mathrm{SiO}_{2}$ (15 to $\left.25 \mathrm{\mu m}\right)$.

$\mathrm{Ca} \cdot\left(\mathrm{Al}_{2} \mathrm{O}_{3}\right)_{y} \cdot \mathrm{SiO}_{2} \cdot \quad(\sim 25 \mu \mathrm{m})$.

$(\mathrm{CaO})_{x} \cdot\left(\mathrm{Al}_{2} \mathrm{O}_{3}\right)_{y}$ (Hexagonal, $\left.15-45 \mathrm{\mu m}\right)$.

$(\mathrm{CaO})_{x} \cdot\left(\mathrm{Al}_{2} \mathrm{O}_{3}\right)$ y (very irregular shape, $15 \mathrm{\mu m}$ ).

$\mathrm{SiO}_{2}(\cdots, 8 \mu \mathrm{m})$.

MnO. FeO $(3-9 \mu \mathrm{m})$.

$(\mathrm{MgO})_{x} \cdot\left(\mathrm{Al}_{2} \mathrm{O}_{3}\right)_{y}$. MnO $(2-4 \mu \mathrm{m})$

Mixtures of $\mathrm{MnS}$ and $\mathrm{Al}_{2} \mathrm{O}_{3}(\sim 4 \mathrm{um})$.

Cu particles $(\sim 1.3 \mathrm{um})$.

$\mathrm{MnS}$ in the shape of very small spheres $(\sim 0.7 \mu \mathrm{m})$. It was noted that the process of void formation consisted of two parts:
The primary nucleation started during the yielding of the material. Only . $\varepsilon_{p}$ As $0.15-0.22$. Contrary to the primary nucleation, the formation and growth of the secondary voids, around the submicron particles was in sudden bursts and fast. It was thought that this rapid growth was achieved by the increasing strain. In the final phase, voids rapidly enlarged and coalesced as the strain reached the instability point. Thus the actual crack formation process was found to be dependent on the instability strain. The formation of primary voids occurred mostly around large inclusions, such as $\mathrm{MnS}$, CaS, CaO. $\mathrm{Al}_{2} \mathrm{O}_{3}$, or $\mathrm{CaO} . \mathrm{MgO} . \mathrm{SiO}_{2}$, Fig. 2. The secondary voids were adjacent to the sub
to the medium size inclusions.

Figure 3 shows the relationship between the relative void volume $V v$ and plastic strain $\varepsilon_{p}$.

The average value of the slope in the strain region limited by $\left(0.15<\varepsilon_{p}<1.0\right)$ is

$$
S=\frac{\ln \left(V_{v} / V_{0}\right)}{\bar{\varepsilon}_{p}-\bar{\varepsilon}_{0}} \simeq 2.2
$$

which represents the value of $\left(1 n V_{v} / V_{0}\right) /\left(\varepsilon_{p}-\varepsilon_{0}\right)$ being nearly constant for smooth cylindrical tensile specimens after necking within the range of $\frac{\sigma_{m}}{\bar{\sigma}} \approx 0.34$ to 0.74 . Two specific values of plastic strain are of interest. Firstly, at $\varepsilon_{p}$ of
0.22 a large number of voids suddenly formed, increasing substantially the relative void volume. We consider this strain being a typical value

corresponding to the beginning of the necking process. The second volume increase occurred at $\varepsilon_{p}$ equal 0.97 which is the instability strain. At increase occurred at $p$ equal fractured.

THE INSTABILITY STRAIN

In the previous paper (1) it was shown that the instability $\operatorname{strain} \varepsilon i$ was In the previous paper (1) it was shown that the instabilaty strain $e_{i}$ was invariably lower than the true fracture strainstability tests giving in ding groups of line instability strain $\varepsilon_{i}$ being tests were performed, in amounting to 1.08 . In the second group both the mean instability strain and the true fracture strain reached higher values, but their ratio was still equivalent to $90 \%$

Rice (15) suggested that the instability strain may be expressed as

$$
\varepsilon_{i}=\sqrt{(1+3 n)(1-n) / 3}
$$

where $n$ is the strain hardening exponent. For our steel 50D, the value o $n$ at instability is 0.27 and using equation (9) we obtain $\varepsilon_{i}=0.664$. This is much lower than the experimental value mentioned above. Similar. but again rather low results were obtained using the Hutchinson's (6) analysis. It should be noted that the strain hardening exponent will 
$n=0.15, \varepsilon_{i}$ was 0.64 , but for the increased $n$ of 0.22 , corresponding to the situation just before necking, $\varepsilon_{i}$ increased only to 0.66 , still very

different from the experimental value. Consequently Equation (9) may need some adjustments. Also, in the true strain range $\varepsilon_{p}=0.5$ to 0.6 number of strings of voids and their coalescence in the loading direction was observed. Because of the low strain and unexpected direction of strin formation, this phenomenon warrants additional investigation.

THE CORRELATION OF TENSILE PROPERTIES AND FRACTURE TOUGHNESS

The relationship between the toughness and some other properties of materials has been investigated by many workers. It is now generally parameters exists. For example, Hahn and Rosenfield (7) suggested that the toughness K1c may be expressed as

$$
K_{1 c}=\left(\frac{2}{3} E \sigma_{y} n^{2} \varepsilon_{f}\right)^{\frac{1}{2}}
$$

This relationship has been successfully used for high strength alloy steels; $i t$ is also suitable for the 50D steel. It is customary to express $J 1 c$ as follows : -

$$
J_{1 c}=\frac{K}{E} c_{c}^{2}\left(1-v^{2}\right)
$$

and applying equation (10) we obtain (adjusted for S1 units)

$$
J_{1 c}=\left(\frac{2}{3}\left(1-v^{2}\right) \quad \sigma_{y} \varepsilon_{f}\right) 25.4
$$

Using the data from $(1,8)$ we finally obtain

$$
J_{1 c}=293 \mathrm{~N} / \mathrm{mm}
$$

\begin{tabular}{|c|c|c|}
\hline or the first group & $:$ & $J_{1 c}=289 \mathrm{~N} / \mathrm{mm}$ \\
\hline the $s$ & $:$ & $J_{1 c}=271 \mathrm{~N} / \mathrm{mm}$ \\
\hline ving a mean va & $:$ & $J_{1 c}=280 \mathrm{~N} / \mathrm{mm}$ \\
\hline
\end{tabular}

Two sets of experiments, using CT-specimens made from two plates, as supplied, were performed according to ASTM E813-81. The following results were obtained:

The difference between the two series of tests amounts to 58 , which is considered satisfactory.

\section{CONCLUSIONS}

The tearing instability concept and the tearing modulus criterion were used to investigate the tensile deformation of the low-alloy steel BS4360-50D in the laboratory air at $200^{\circ} \mathrm{C}$, applying a loading rate of $0.05 \mathrm{~mm} / \mathrm{min}$. The nucleation of voids was studied in detail.

1. The primary voids nucleated around larger $(>10 \mu \mathrm{m})$ inclusions, such as $\mathrm{MnS}, \mathrm{CaS}$ and $\mathrm{CaO} . \mathrm{Al}_{2} \mathrm{O}_{3}$. $\mathrm{SiO}_{2}$. At much higher strains an additional nucleation of secondary voids occured even at very small inclusions $(<1 \mu \mathrm{m})$.
A linear pline strain range 0.22 to 0.97 .

. platic strain was obs the true The instability strain for this steel was abouthe theoretically fracture strain.
derived $\operatorname{strain} \varepsilon_{i}=0.66$.

4. The estinated toughness $J_{10}=293 \mathrm{~N} / \mathrm{mm}$ derived from tensile data was The estimated toun multiple specimens method, ASTM E 813-81.

\section{REFERENCES}

(1) Zheng, C.Q. and Radon, J.C (1983). Basic tensile properties of low-alloy steel BS4360-50D. Proc. Conf. Fracture Mechanics Technology applied to material evaluation and structure desig Melbourne, Australia, J.C. Sih; N.E. Royan

M. Nijhoff, Publishers, Elastic-Plastic Fracture, ASTM STP 668 $5-36$.

(1982). Application of Elastic Plastic Fracture Mechanics to a steel in the ductile brittle transition region, M.Sc. Thesis,

4) Kong, B. and Paris, P.C. (1979). Fracture Mechanics, ASTM STP 677, $770-780$.

(5) Needleman, and Rice, J.R. (1978). Limits to ductility set by Plastic flow Plastic flow localization, Mechaties, 237-267.

(6) General Motors Research Laboratories, 61-71.

7) Hahn, G.T. and Rosenfield, A.K. (1968). App

8) (7hen ) . The formation of voids in th ductile ductile fracture of a lijing), Science pres, Beijing, 1052-1056.

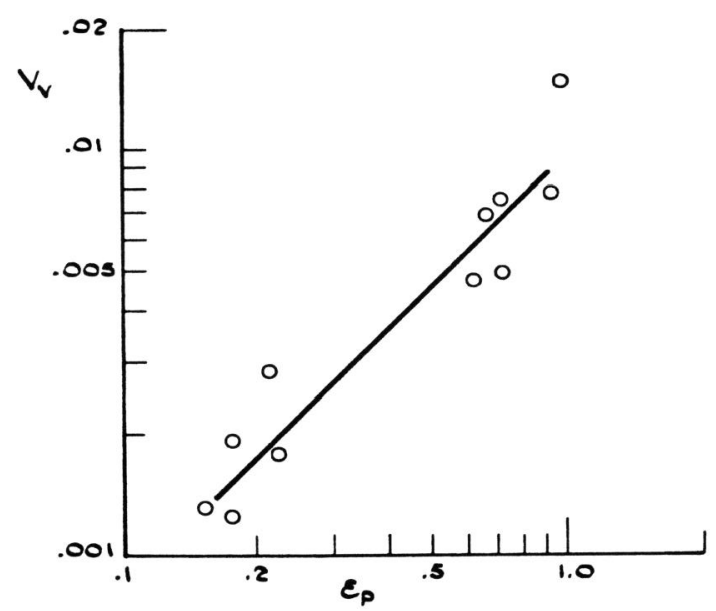

ig. 3. Relative void volume $v$ vs. plastic strain. 

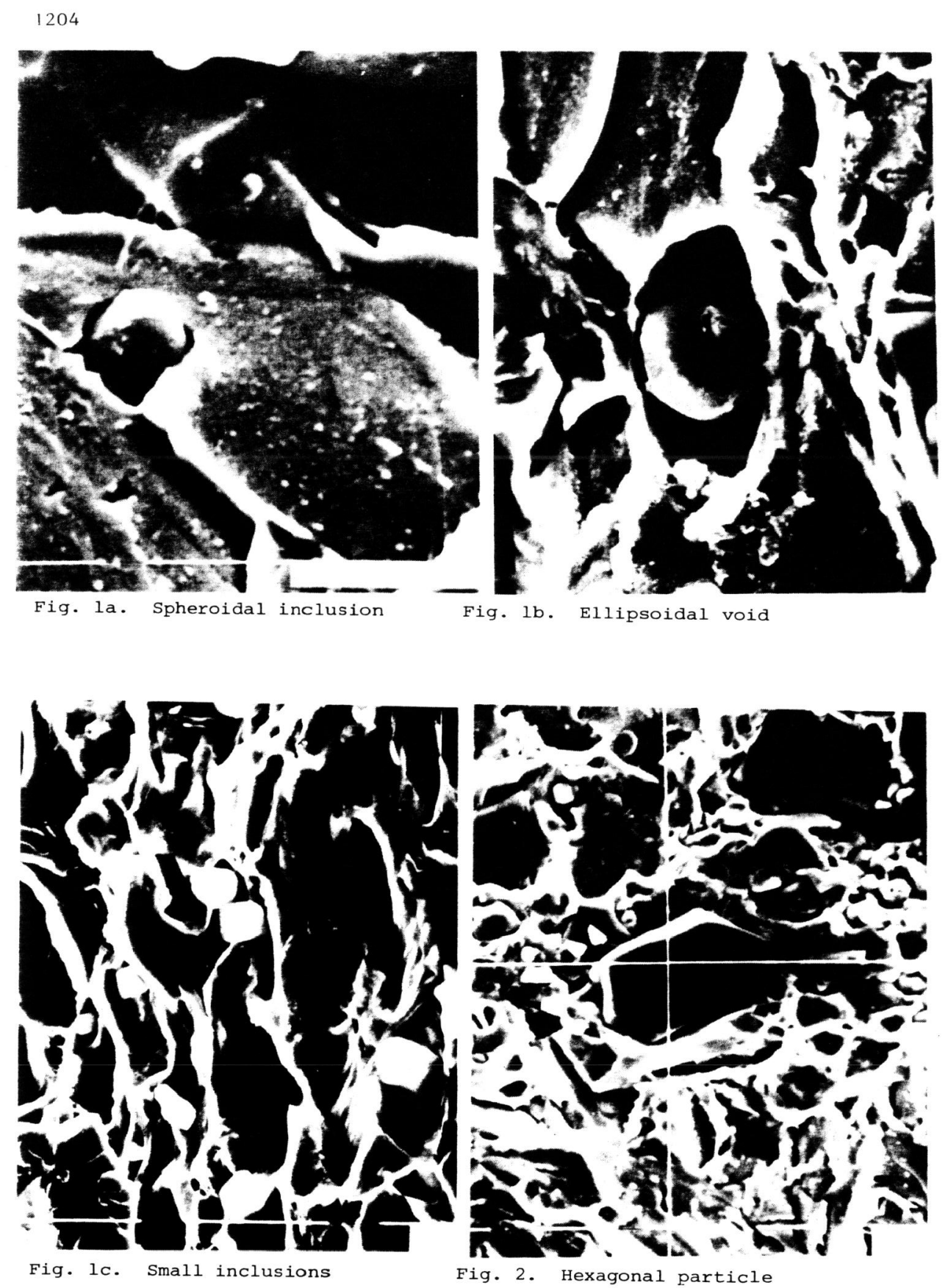